\title{
Comparison of whole-tree wood property maps based on near-infrared spectroscopic calibrations utilizing data at different spatial resolutions
}

https://doi.org/10.1515/hf-2019-0026

Received January 31, 2019; accepted July 12, 2019; previously published online August 15, 2019

\begin{abstract}
Near-infrared (NIR) spectra or NIR-hyperspectral images obtained from radial strips or wood discs provide a cost-effective methodology for examining wood property variation within trees. The calibration used for wood property prediction is critical and can be obtained using two fundamentally different approaches. One involves using a spatial-specific model where wood property data and corresponding spectral data are measured at the same resolution for calibration and prediction, e.g. 10 -mm radial increments. The other provides a spatialinterpolated model and involves measuring a property on a broad-scale, e.g. whole-tree, calibrating this data against NIR spectra representing the equivalent scale and then using the calibration to predict the property at higher resolution. To understand the impact of these approaches on subsequent patterns of within-tree variation, wholetree air-dry density (ADD) and coarseness maps, based on data obtained using the two different approaches, were compared. Patterns of ADD and coarseness variation were comparable indicating that both approaches can be utilized to examine within-tree variation. Spatialinterpolated models have a distinct advantage; being based on whole-tree (or disc) samples, they greatly reduce the cost of wood property analysis and allow the development of maps for properties that are costly and difficult to measure, for example, pulp yield.
\end{abstract}

Keywords: air-dry density, coarseness, loblolly pine, nearinfrared spectroscopy, Pinus taeda, SilviScan, within-tree variation, wood property maps

\footnotetext{
*Corresponding author: Laurence R. Schimleck, Department of Wood Science and Engineering, College of Forestry, Oregon State University, Corvallis, OR 97331, USA, e-mail: Laurence.Schimleck@oregonstate.edu. https://orcid.org/0000-0003-1958-6059

Finto Antony and Joseph Dahlen: Warnell School of Forestry and Natural Resources, University of Georgia, 180 E. Green Street, Athens, GA 30602, USA

Christian Mora: Fibraconsult, Concepcion 4030000, Chile
}

\section{Introduction}

Our knowledge of the within-tree variation of most wood properties is extremely limited (Burdon et al. 2004) owing to the time and cost involved in measuring the necessarily large number of samples required to describe radial and longitudinal variation within trees accurately. In fact, the measurement of many properties is such that any attempt to create a map of a within-tree variation is impractical using standard measurement techniques.

SilviScan and near-infrared (NIR) spectroscopy have provided data that have allowed the examination of withintree variation at a level of detail and a degree of certainty that until recently was impossible (Evans et al. 2000; Mora and Schimleck 2009). SilviScan measures a range of properties utilizing a combination of image analysis (tracheid properties), X-ray densitometry (density) and X-ray diffractometry [microfibril angle (MFA) and stiffness (modulus of elasticity [MOE])] (Evans 1994, 1999, 2006) at high spatial resolution and has been used to develop detailed maps for both hardwoods and softwoods. NIR spectroscopy can estimate a wide range of wood properties; but before it can be used to examine the within-tree variation for any property, a model must be developed which is subsequently used for wood property prediction. NIR-based calibrations for properties measured by SilviScan have been reported (Schimleck et al. 2001; Schimleck and Evans 2004), and NIR calibrated with SilviScan data has been used to examine the within-tree variation for a range of properties in loblolly pine (Pinus taeda L.) (Mora and Schimleck 2009; Schimleck et al. 2018a). While NIR spectroscopy may be viewed as a cost-saving approach compared to SilviScan, it also provides a complementary technique as it can be used to estimate several properties (tracheid length, pulp yield, lignin, cellulose) that SilviScan cannot measure. Examples of maps of within-tree variation using NIR estimation of wood properties complimentary to SilviScan include pulp yield in shining gum (Eucalyptus nitens $\mathrm{H}$. Dean \& Maiden) (Schimleck and Michell 1998) and tracheid length in loblolly pine (Lundqvist et al. 2005).

The calibration used in NIR-based studies is critical to the success of the method. Recent studies of within-tree 
variation (Mora and Schimleck 2009; Thumm et al. 2010; Thumm et al. 2016; Schimleck et al. 2018a,b) have seen two general approaches used for calibration development. The first involves the utilization of a spatial-specific model where wood property data and the corresponding spectral data are at the same spatial resolution for both calibration and prediction. An example of a spatial-specific model is where radial strip samples are cut from pith to bark, and NIR measurement and the reference data are collected at the same scale, such as in 10-mm radial increments (Mora and Schimleck 2009; Nabavi et al. 2018; Schimleck et al. 2018a).

The second approach provides a spatial-interpolated model and involves measuring a property on a broadscale, e.g. whole-disc or whole-tree, calibrating this data against NIR spectra representing the equivalent scale and then using the calibration to predict the property at much higher resolution (Thumm et al. 2010, 2016; Schimleck et al. 2018b). The second approach has been frequently reported in studies that have utilized NIR hyperspectral imaging (NIR-HSI) to examine the variation in wood properties over the cross-sectional surface of discs (Thumm et al. 2010, 2016), lumber (Schimleck et al. 2018b) and the radial-longitudinal surface of radial strips (Ma et al. 2017, 2018). NIR-HSI is an emerging technology that "combines spectroscopy and imaging resulting in three dimensional multivariate data structures ('hypercubes'). Each pixel in a hypercube contains a spectrum representing its light-absorbing and scattering properties. This spectrum can be used to estimate chemical composition and/or physical properties of the spatial region represented by that pixel" (Burger and Gowen 2011). As it is not feasible to measure wood properties at the same resolution as pixels in an image, it has been necessary to average the pixels/spectra to match the measured wood property. In studies where this approach has been used, properties were measured at the disc level (Thumm et al. 2010, 2016) and for whole pieces of lumber (Schimleck et al. 2018b).

In the case whereby NIR or NIR-HSI data are collected from radial strips but wood property data are collected at the whole-disc or whole-tree level, there is a mismatch between the radial increment and the wood property as the increment actually represents an area (disc measurement) or volume (whole-tree measurement). The reason for the mismatch is that the collection of NIR spectra using traditional instruments from radial strips is routine (Nabavi et al. 2018, etc.), while the collection of disc-level NIR spectra is not. Thus, the NIR spectra within a specific radial section, such as $10 \mathrm{~mm}$, should be weighted by the appropriate basal area to better reflect the within-tree variation. The impact of the two approaches on patterns of within-tree variation (or variation over a cross-sectional surface) has not been investigated, and the aim of this study was to compare whole-tree air-dry density (ADD) and coarseness maps based on data obtained using the two different approaches.

\section{Materials and methods}

Sample origin: Two sets of $18 P$. taeda trees, aged 13 and 22 years, respectively, were sampled from a half-sib progeny trial planted at the International Paper's (IP) Southlands facility located near Bainbridge, GA, USA. The trees were a subsample of two larger populations selected to encompass the range of cellulose, lignin and specific gravity (SG) variation as measured by IP. Selected trees were felled and samples removed for wood property and NIR analyses. Four bolts ( $0.75 \mathrm{~m}$ long) per tree with each bolt cut in the center of each $25 \%$ of total height and discs ( $25 \mathrm{~mm}$ thick) were taken at $1.5-\mathrm{m}$ intervals along the stem of each tree, giving 9-13 discs per tree (number depended on tree height). A total of 191 discs were collected from trees aged 13 years, while 220 were obtained from the 22-year-old trees, giving a total of 411 discs.

Sample preparation - radial strips: Pith-to-bark radial sections were cut from each disc using a bandsaw. Section dimensions were $12.5 \mathrm{~mm}$ longitudinally by $12.5 \mathrm{~mm}$ tangentially with the radial length corresponding to the radius of the disc. Radial sections were gently dried, glued into core holders and cut using a twin-blade saw to give strips $2 \mathrm{~mm}$ thick (tangentially) using the methodology described in Jordan et al. (2008).

Near-infrared spectroscopy: Schimleck et al. (2009) provided a detailed description of the methodology used to collect NIR spectra. Briefly, NIR spectra were collected in $10-\mathrm{mm}$ radial increments from the radial-longitudinal face of each radial strip using a FOSS NIRSystems Inc. (Laurel, MD, USA) Model 5000 scanning spectrometer. The spectra were collected at 2-nm intervals over the wavelength range of $1100-2500 \mathrm{~nm}$. The total number of radial spectra collected was 2569 (1114 from 13-year-old trees and 1455 from trees aged 22 years).

Wood property measurement: A subsample of 72 strips (two from each tree and representing different heights) was selected for SilviScan determination of ADD, MFA, MOE and several tracheid properties including coarseness (Evans 1994, 1999, 2006). From the same trees, four 0.75-m bolts were chipped using a 1.2-m Carthage chipper (Carthage Machine Company, Carthage, NY, USA) at the Institute of Paper Science and Technology (IPST), Atlanta, GA, and then thoroughly mixed to give a single composite sample per tree. Though several properties were measured for this study, our focus was on density and tracheid coarseness as they were the only properties measured on both radial strips and whole-tree samples.

The basic density (oven-dry weight, green volume) was measured on the whole-tree chip samples by water displacement and by oven-drying the chips (White et al. 2009). White et al. (2009) provided a detailed description of pulping. Briefly, for each whole-tree 
sample, duplicate cooks were performed on $1 \mathrm{~kg}$ of oven-dried whole-tree chips in a 10-1 M/K digester. Two pulp grades (linerboard at $100 \kappa$ and bleachable at $30 \kappa)$ were obtained for each sample. For both pulp grades, the fiber (tracheid) coarseness was measured using a fiber quality analyzer (FQA) (OpTest Equipment Inc., Hawkesbury, Canada) (White et al. 2011); however, in this study, only the fiber coarseness for the linerboard pulps were used.

The measured wood properties (radial strips vs. whole-tree chips or pulp) were adjusted to a common basis to facilitate comparisons between them. The basic density values of the chips were converted to a SilviScan equivalent ADD which corresponds to a moisture content of $7.7 \%$, and thus was a change from $0 \%$ to $7.7 \%$ moisture for the weight, and a change in the volumetric dimensions of $9 \%$. The $9 \%$ change in dimensions corresponds to a change in volume from the fiber saturation point for loblolly pine, assumed to be $28.7 \%$ moisture content, to the $7.7 \%$ moisture corresponding to SilviScan ADD, using $12.3 \%$ as the total volumetric change for loblolly pine from the fiber saturation point (assumed 28.7\%) to 0\% for loblolly pine (Glass and Zelinka 2010).

Adjusting densities to a comparative basis is routine, but the same cannot be said for coarseness as during pulping, the tracheid width and wall thickness decrease due to the loss of hemicellulose and lignin (Scallan and Green 1975; Kibblewhite and Evans 2001). Reductions in tracheid dimensions change pulp coarseness and are related to reductions in pulp yield (Çöpür et al. 2005). To the authors' knowledge, models for adjusting coarseness are not available in the literature for loblolly pine, so we used the radiata pine data presented in Kibblewhite and Evans (2001). Specifically, this paper does not present model parameters, but data for the individual trees are presented and thus utilized here to construct a linear model to adjust from coarseness measured on pulped fibers to coarseness measured on dry samples using SilviScan. The adjustment from pulped coarseness to dry coarseness is as follows:

$$
\text { CoarsenessDry }=210+1.0062 * \text { CoarsenessPulp }
$$

where CoarsenessDry is the coarseness predicted on dry solid samples using SilviScan, and CoarsenessPulp is the coarseness measured on the pulp obtained from the kraft process (Kibblewhite and Evans 2001).

ADD and coarseness calibrations: Two distinct model types (spatial-specific and spatial-interpolated) were examined in this study. For the spatial-specific models (referred to in the following text as NIR-SilviScan models), SilviScan ADD and coarseness data were averaged over 10-mm sections from pith to bark for use in model building with the 10-mm NIR spectra data (477 in total, representing 179 and 298 spectra from trees aged 13- and 22-years, respectively) (Schimleck et al. 2018a). The 10-mm spatial-specific model was then used to predict ADD and coarseness for all 2569 spectra in $10-\mathrm{mm}$ increments.

Spatial-interpolated models were developed at the whole-tree level. This required that the $10-\mathrm{mm}$ spectral data from each tree be averaged to give a single representative spectrum (2569 10-mm increment spectra averaged to 36 in total) to allow for model building with the wood properties measured at the whole-tree level. Two approaches were used to determine the average tree spectra, with the first being an arithmetic average (NIR models based on these spectra are referred to in the text as NIR-Mean) for all spectra within a tree. The second was a basal-area weighted average (NIR models $=$ NIR-Weighted) which weighed spectra based on the basal area they represented within the tree. For each wavelength from 1100 to $2500 \mathrm{~nm}$ in 2-nm intervals, the following steps were conducted. First, the basal area for each radial position represented within a disc was calculated as follows:

$$
\text { area }=\pi r_{o}^{2}-\pi r_{i}^{2}
$$

where are $a$ is the area that a specific $10-\mathrm{mm}$ radial section represents within a disc, $r_{o}$ is the outside radius of the $10-\mathrm{mm}$ section, and $r_{i}$ is the inside radius of the $10-\mathrm{mm}$ section. Note that NIR spectra at the bark represent a greater area than NIR spectra at the pith, because their area is larger and thus they are given a larger weight within the radial strip. The weighted average spectrum for each disc was then calculated as follows:

$$
\text { spectrum }_{\text {disc }}=\sum_{i=1}^{n} \frac{\text { area }_{i} * \text { spectrum }_{i}}{\text { area }_{\text {disc }}}
$$

where spectrum ${ }_{\text {disc }}$ is the weighted average spectra for a specific disc, area $_{i}$ is the area that a specific $10-\mathrm{mm}$ radial section represents within a disc, spectrum $_{i}$ is a specific spectrum, area $_{\text {disc }}$ is the total area of a disc, and $\mathrm{n}$ is the number of $10-\mathrm{mm}$ radial sections within each disc. The weighted average spectrum for each tree was then calculated as follows:

$$
\text { spectrum }_{\text {tree }}=\sum_{i=1}^{k} \frac{\text { area }_{\text {disci }} * \text { spectrum }_{\text {disci }}}{\text { area }_{\text {tree }}}
$$

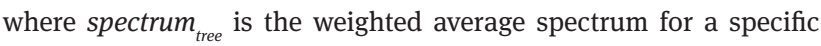
tree, are $_{\text {disci }}$ is the area that a specific disc represents, spectra $_{\text {disci }}$ is the calculated spectra for each disc, area $_{\text {tree }}$ is the total area for each tree, and $k$ is the number of discs within each tree. Note that the weighted average NIR spectra at lower height levels within each tree (such as the stump) represent a greater amount of the total area of the discs than the NIR spectra at the tip of the tree.

Untreated spectral data, as used by Schimleck et al. (2009) and Mora and Schimleck (2009), were used to create the calibrations using partial least squares (PLS) regression. NIR-SilviScan calibrations were developed with four cross-validation segments while calibrations for the whole-tree data (NIR-Mean and NIR-Weighted average) were developed using leave-one-out cross-validation. The calibration performance was assessed using the following parameters:

- Standard error of calibration (SEC) determined from the residuals of the final calibration;

- Standard error of cross-validation (SECV) determined from the residuals of each cross-validation phase;

- Coefficient of determination $\left(R^{2}\right)$, the proportion of variation in the calibration set that was explained by the calibration;

- $\quad$ Ratio of performance to deviation (RPD) (Williams and Sobering 1993), calculated as the ratio of the standard deviation of the reference data to the SECV.

Determination of RPD allows comparison of calibrations for different properties that have differing data ranges and units; the higher the RPD, the more accurate the data is described by the calibration or predicted. The PLS models were then used to predict ADD and coarseness for all 2569 spectra in 10-mm increments. Figure 1 provides a summary of the three models.

Maps of wood property variation within trees: Mora and Schimleck (2009) provided a detailed description of the methodologies 


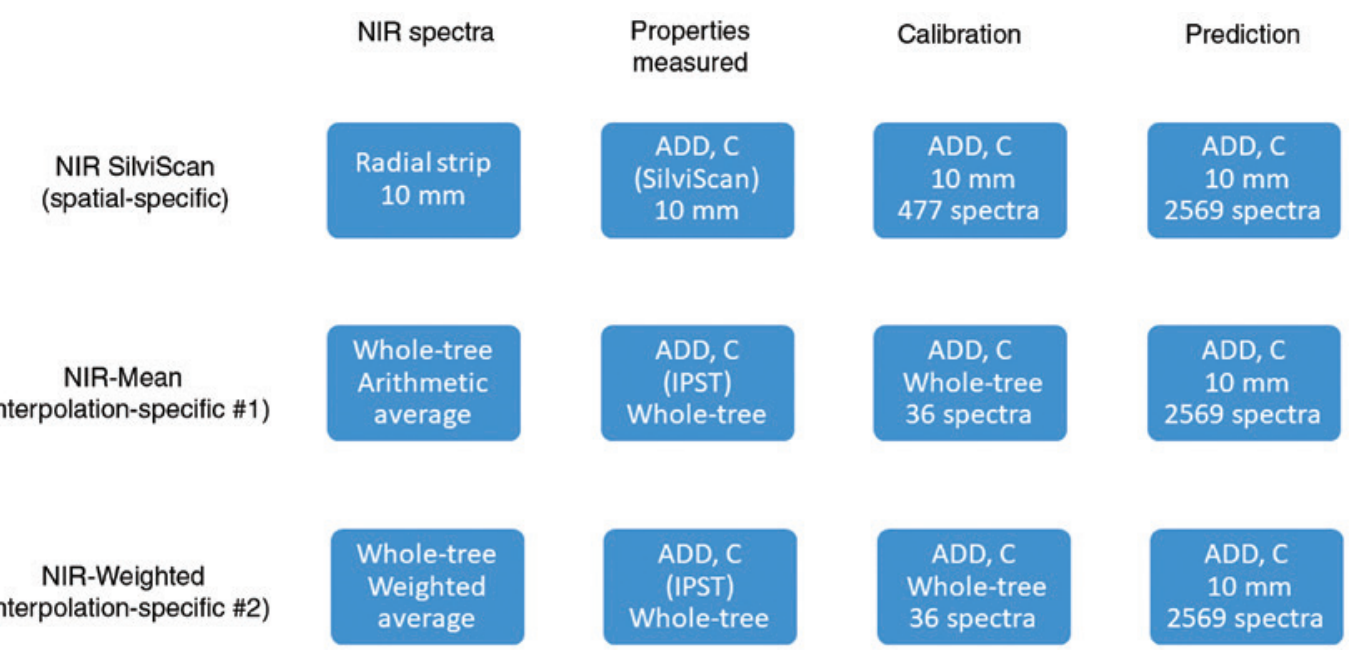

Figure 1: Summary of the three different models developed for air-dry density (ADD) and tracheid coarseness (C).

they used to develop maps of within-tree variation for ADD and MFA. Three different methods were explored: Akima's interpolation, Universal Kriging and semiparametric smoothing. Mora and Schimleck (2009) reported that for loblolly pine, maps generated by Akima's interpolation method provided a good representation of the expected trends in ADD and MFA. In addition, they reported that a principal advantage of Akima's algorithm over the Universal Kriging and semiparametric smoothing techniques was that only straightforward procedures were required, and there were no problems concerning computational stability or convergence. Owing to these features, only Akima's interpolation method was used to provide the maps that were used to compare spatial-specific (NIRSilviScan) and spatial-interpolated (NIR-Mean, NIR-Weighted) models. Maps for the 13- and 22-year-old trees were built using data predicted by the NIR-SilviScan, -Mean and -Weighted average approaches.

Comparison between predictions and measured data: For each age, probability density plots (where the area under the density curves has a probability of 1 ) were produced to show the variation in the predicted properties for the three different sets of ADD and coarseness values. The predicted NIR-SilviScan, NIR-Mean and NIR-Weighted average prediction values were compared for ADD and coarseness using a linear model to determine the model accuracy between the three methods. The whole-tree mean density and coarseness were calculated for each of the three methods by weighting the volume of the point compared to the overall volume. The weighted property was then summed up for each respective height and radial position combination to yield the mean ADD and coarseness of each tree.

Data analysis: All data were analyzed using the R statistical programing environment (R Core Team 2018) with the RStudio interface (RStudio 2018) and the tidyverse series of packages (Wickham and RStudio 2017). The NIR calibration models were developed using the pls (Mevik et al. 2013) and signal (Signal Developers 2013) packages. Mean and weighted spectra were calculated using the dplyr package (Wickham and Francois 2016). The ADD and coarseness within-tree variation maps (for the trees aged 13- and 22-years) were produced using the akima (Akima and Gebhardt 2016), fields (Nychka et al. 2015) and lattice (Sarkar 2008) packages.

\section{Results and discussion}

\section{Wood property calibrations}

The NIR-SilviScan ADD calibration, developed using 10-mm SilviScan ADD data and NIR spectra measured in 10-mm increments, and the two spatial-interpolated ADD calibrations (NIR-Mean, NIR-Weighted), developed using whole-tree ADD and NIR data, are summarized in Table 1. The NIR-SilviScan ADD calibration was based on five factors and had a high $\mathrm{R}^{2}(0.88)$ and good $\mathrm{RPD}_{\mathrm{c}}$ (2.8). For the NIR-Mean and NIR-Weighted ADD calibrations, two factors were used and the models had very similar statistics. Calibration statistics were inferior to those reported for the NIR-SilviScan ADD model, but this could be expected as the range of the calibration data for the whole-tree models $\left(462-651 \mathrm{~kg} \mathrm{~m}^{-3}\right)$ was not as large as the SilviScan ADD data (320-912 $\mathrm{kg} \mathrm{m}^{-3}$ ). For coarseness, the NIR-SilviScan calibration also had a high $\mathrm{R}^{2}$ (0.86), while the NIR-Mean and NIR-Weighted models had only one factor recommended and were much weaker $\left(\mathrm{R}^{2}\right.$ for both $=0.42$ ). The narrow range for the FQA whole-tree values for coarseness (443-599 $\mu \mathrm{g} \mathrm{m}^{-1}$ ) compared to the SilviScan values (359-800 $\mu \mathrm{g} \mathrm{m}^{-1}$ ) may explain the inferior models. The reduced range for the whole-tree values compared to the SilviScan values arises as SilviScan values are measured at multiple locations within the tree as opposed to a single composite value. 
Table 1: Summary of the NIR-SilviScan, NIR-Mean and NIR-Weighted loblolly pine wood property calibrations.

\begin{tabular}{|c|c|c|c|c|c|c|c|}
\hline \multirow[b]{2}{*}{ Property } & \multirow[b]{2}{*}{ Model } & \multirow{2}{*}{$\begin{array}{l}\text { Reference data } \\
\text { (number of samples) }\end{array}$} & \multicolumn{5}{|c|}{ Calibration statistics } \\
\hline & & & Factors & $\mathbf{R}^{2}$ & SEC & SECV & $\mathbf{R P D}_{\mathrm{c}}$ \\
\hline \multirow[t]{3}{*}{ Density } & NIR-SilviScan & SilviScan (477) & 5 & 0.88 & 41.8 & 43.0 & 2.8 \\
\hline & NIR-Mean & Whole-tree chips (36) & 2 & 0.69 & 26.9 & 29.2 & 1.8 \\
\hline & NIR-Weighted & Whole-tree chips (36) & 2 & 0.70 & 26.7 & 29.2 & 1.8 \\
\hline \multirow[t]{3}{*}{ Coarseness } & NIR SilviScan & SilviScan (477) & 7 & 0.86 & 42.7 & 44.2 & 2.6 \\
\hline & NIR-Mean & Whole-tree chips (36) & 1 & 0.42 & 29.9 & 31.1 & 1.3 \\
\hline & NIR-Weighted & Whole-tree chips (36) & 1 & 0.42 & 29.9 & 31.0 & 1.3 \\
\hline
\end{tabular}

For the NIR-SilviScan model, samples represented 10-mm sections of radial strips, while for the NIR-Mean and NIR-Weighted models, samples represented individual tree.

NIR, Near-infrared; RPD, ratio of performance to deviation; SEC, standard error of calibration; SECV, standard error of cross-validation.

\section{ADD maps - 13-year-old trees}

Maps showing ADD variation within 13-year-old loblolly pines based on predictions provided by the three different models are shown in Figure 2. In general, all maps are consistent with what is expected for ADD variation within loblolly pine trees (Burdon et al. 2004; Jordan et al.
2008; Schimleck et al. 2018a). They also show very similar patterns of variation; however, the NIR-Weighted spatialinterpolated map had lower estimates of ADD which gave a larger region of low ADD wood near the pith and shifted the high ADD region at the base closer to the periphery of the map. ADD probability (density) plots (Figure 3) are consistent with this observation. Figure 3 also shows that

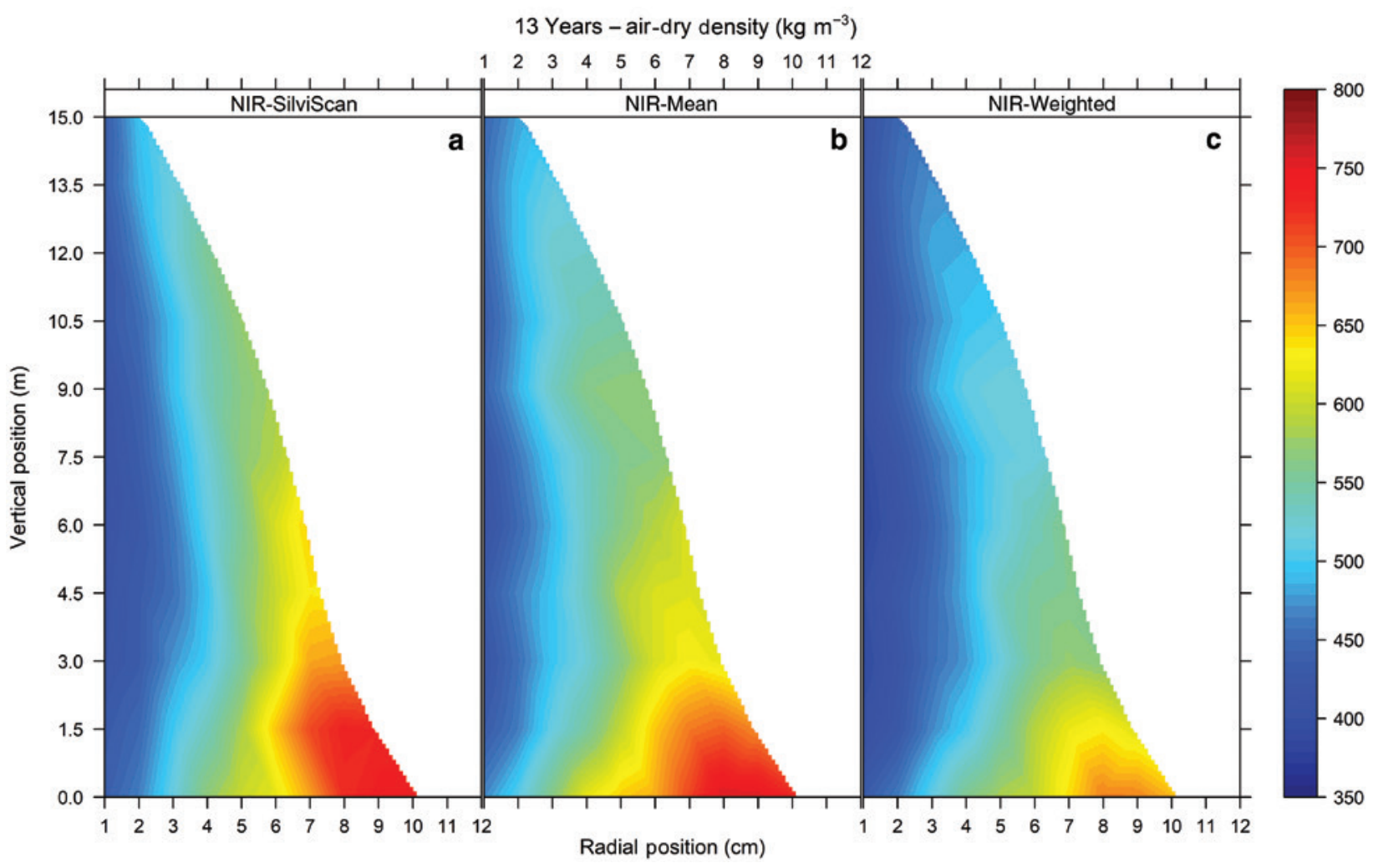

Figure 2: Maps showing within-tree variation of air-dry density (ADD) for loblolly pine trees aged 13 years.

Maps represent the average of 18 trees and were developed using Akima's interpolation method and data provided by (a) spatial-specific ADD model (NIR-SilviScan), (b) interpolation-specific ADD model (NIR-Mean) and (c) interpolation-specific ADD model (NIR-Weighted). 


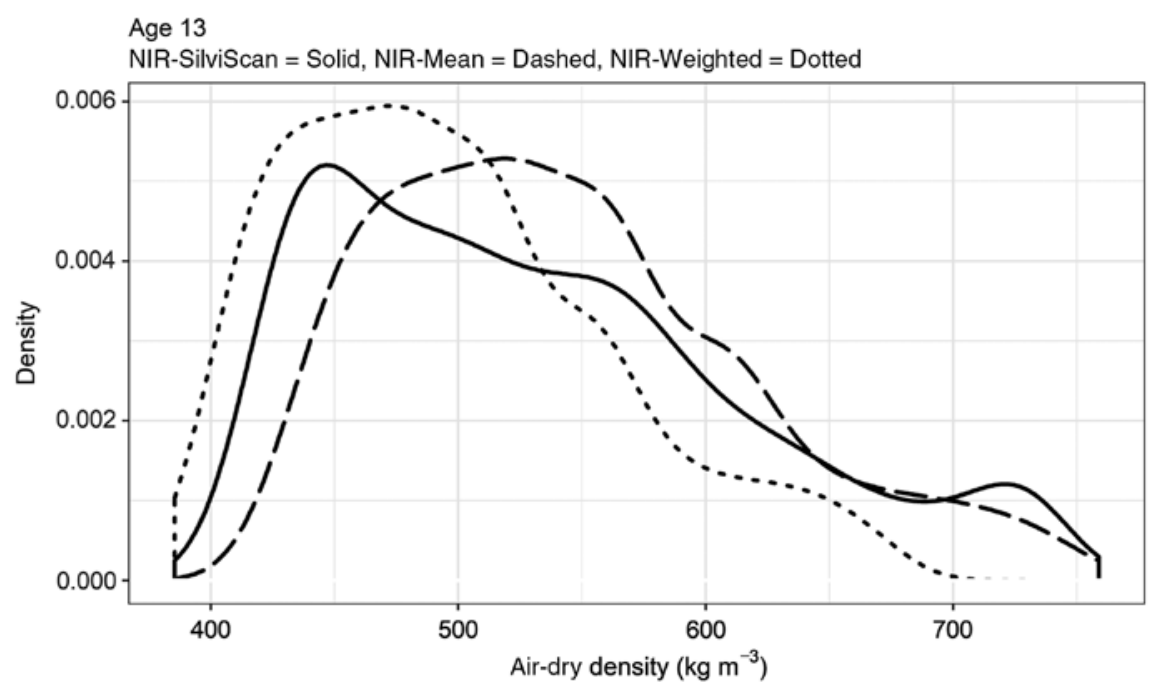

Figure 3: Probability (density) plots showing the variation of predicted density for trees aged 13 years.

the shape of the probability plots based on predicted ADDs from the NIR-Mean and NIR-Weighted models were more similar than the plot based on the NIR-SilviScan spatialspecific model, though for ADD predictions greater than $575 \mathrm{~kg} \mathrm{~m}^{-3}$, the two model types compared very well.

\section{ADD maps - 22-year-old trees}

ADD maps for the 22-year-old trees (Figure 4) showed an increase in ADD at all heights from pith to bark, an observation consistent with maturation (increases in percent

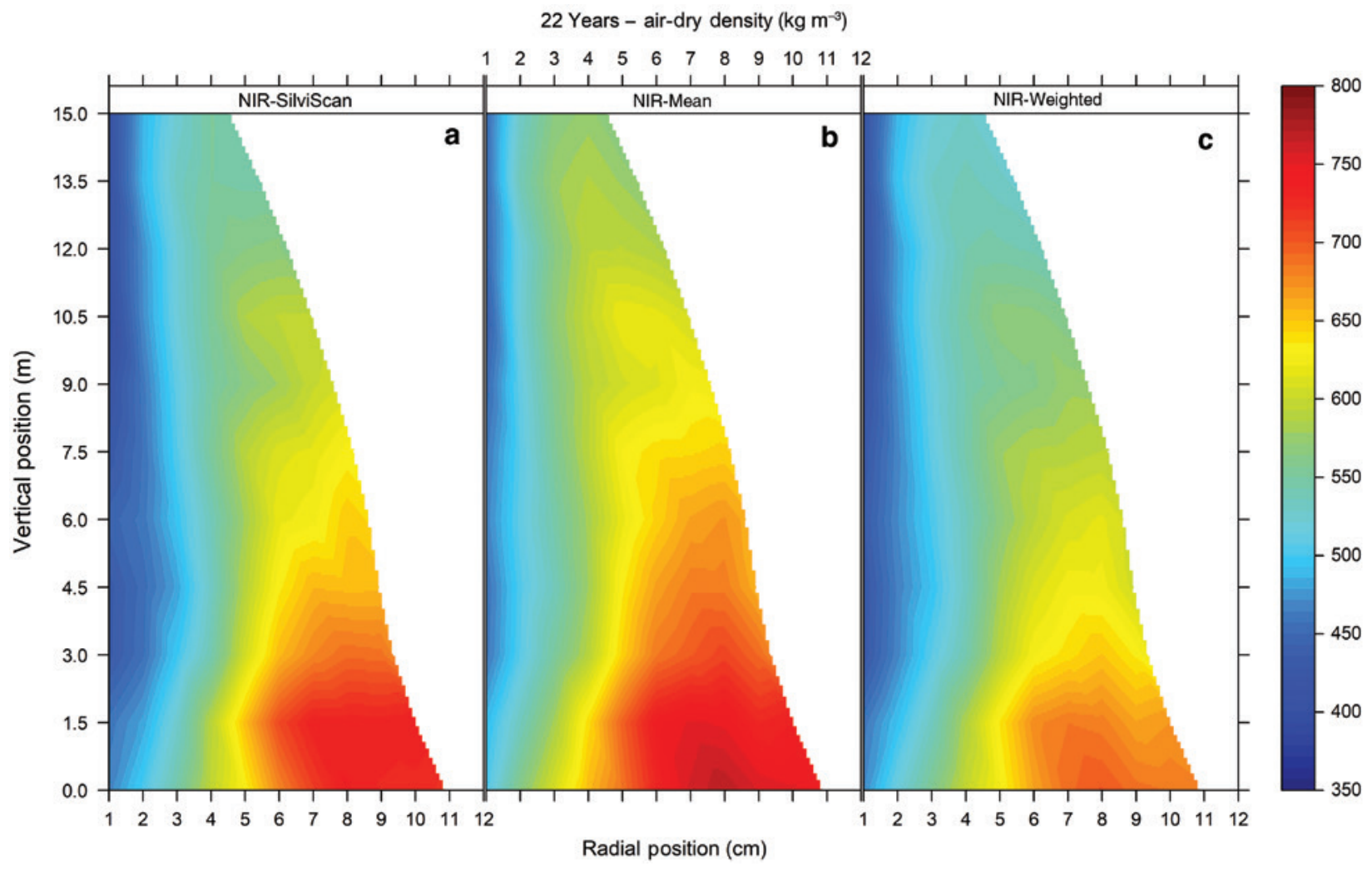

Figure 4: Maps showing within-tree variation of density (ADD) for loblolly pine trees aged 22 years.

Maps represent the average of 18 trees and were developed using Akima's interpolation and data provided by (a) spatial-specific ADD model (NIR-SilviScan), (b) interpolation-specific ADD model (NIR-Mean) and (c) interpolation-specific ADD model (NIR-Weighted). 
latewood and tracheid wall thickness) as loblolly pine trees grow older (Burdon et al. 2004). The lower predictions of ADD for the NIR-Weighted model were again apparent, while the higher ADD predictions of the NIRMean model were more pronounced compared to the maps for the 13-year-old trees, with a zone of very high density at the base of the tree. The probability plots (Figure 5) demonstrated the age-related shifts in ADD (also observed by Schimleck et al. 2009) and the tendency toward higher ADD predictions by the NIR-Mean model. While the maps were similar, the two spatialinterpolated models (NIR-Mean, NIR-Weighted) did not match the range of predictions provided by the spatialspecific (NIR-SilviScan) model, with the NIR-Mean and NIR-Weighted models tending to over- and under-predict the ADD, respectively.

\section{Coarseness maps - 13-year-old trees}

Overall, the pattern of coarseness variation (Figure 6) was consistent with the maps for ADD, i.e. both properties demonstrate a radial increase at all heights (Burdon et al. 2004). However, the coarseness maps were not as consistent between the models as the ADD maps owing to the variable changes that occur in tracheid coarseness during pulping (Scallan and Green 1975; Kibblewhite and Evans 2001; Çöpür et al. 2005). The NIR-SilviScan coarseness map shows greater within-tree variation compared to the NIR-Mean and NIR-Weighted spatial-interpolated maps which do not have coarseness values below $425 \mu \mathrm{g} \mathrm{m}^{-1}$. Probability plots (Figure 7) show NIR-SilviScan predictions had a much larger range than the NIR-Mean and NIR-Weighted approaches and confirm that the three sets of predictions were less consistent than for ADD.

\section{Coarseness maps - 22-year-old trees}

Coarseness maps for the 22-year-old trees showed again the radial increase of coarseness as cambial age increases (Figure 8). Compared to the 13-year-old tree maps, there was an increase in the coarseness values at or greater than $600 \mu \mathrm{g} \mathrm{m}^{-1}$ with the NIR-SilviScan map (spatial-specific) having the highest coarseness values. The probability plots (Figure 9) demonstrated the age-related shifts in coarseness. The distributions were more similar but as with the 13-year-old trees, the NIR-SilviScan model provided data with a wider range.

When the predicted values for the NIR-SilviScan model were plotted against the NIR-Mean and NIR-Weighted models (Figure 10), the datasets agreed very well for the ADD, with both models having a coefficient of determination $\left(\mathrm{R}^{2}\right)$ equal to or greater than 0.90 . The tendency of the NIR-Mean model to over-predict the ADD was noticeable over the range of ADD values, while the under-predictions of the NIR-Weighted model were only apparent for densities greater than $550-600 \mathrm{~kg} \mathrm{~m}^{-3}$. Overall differences in predictions were slightly smaller for the NIR-Weighted model [root mean square error $\left(\right.$ RMSE) $=32.3 \mathrm{~kg} \mathrm{~m}^{-3}$ compared to $32.6 \mathrm{~kg} \mathrm{~m}^{-3}$. There was a much higher deviation between the NIR-SilviScan model and the NIR-Mean and NIR-Weighted models for predicted coarseness compared

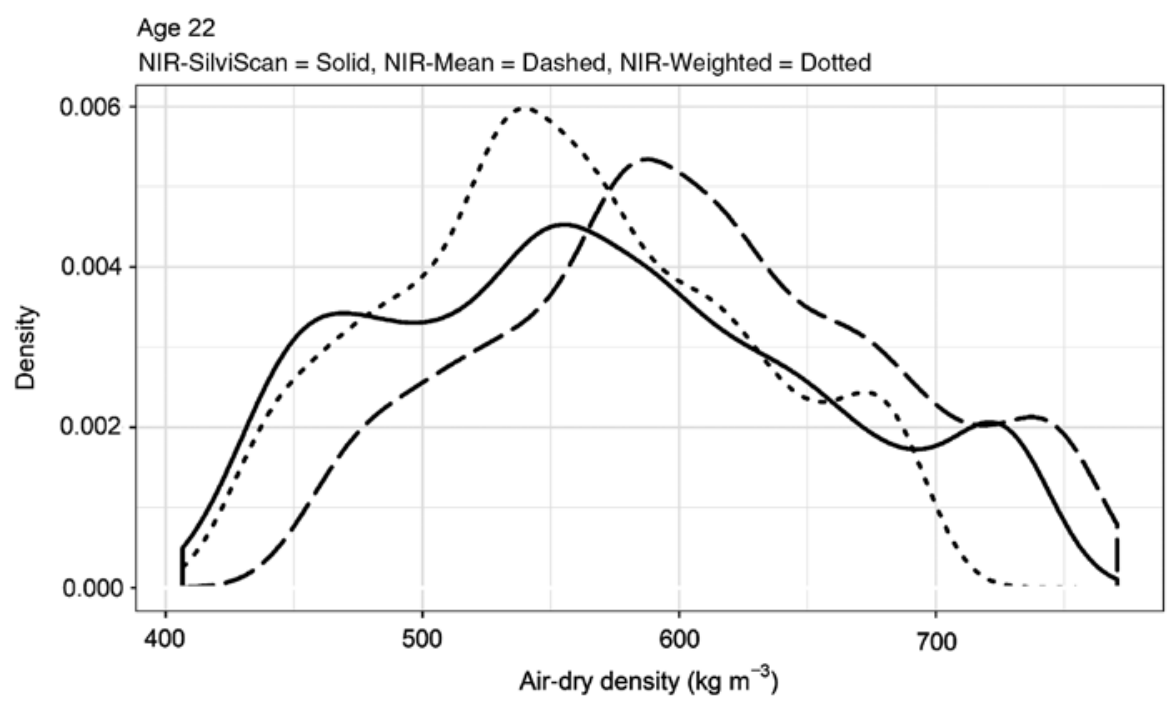

Figure 5: Probability (density) plots showing the variation of predicted density for trees aged 22 years. 


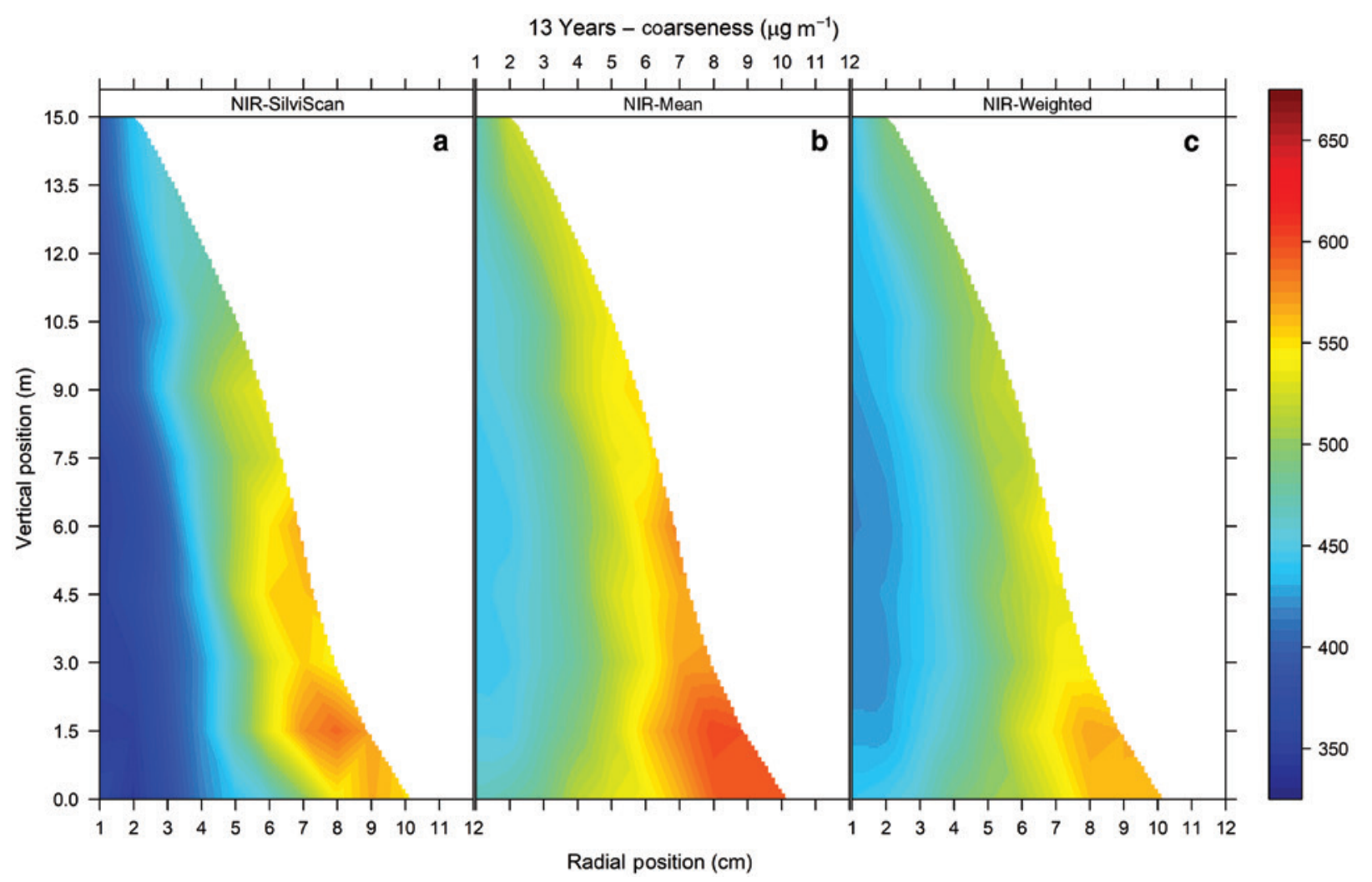

Figure 6: Maps showing within-tree variation of tracheid coarseness for loblolly pine trees aged 13 years.

Maps represent the average of 18 trees and were developed using Akima's interpolation and data provided by (a) spatial-specific coarseness model (NIR-SilviScan), (b) interpolation-specific coarseness model (NIR-Mean) and (c) interpolation-specific coarseness model (NIR-Weighted).

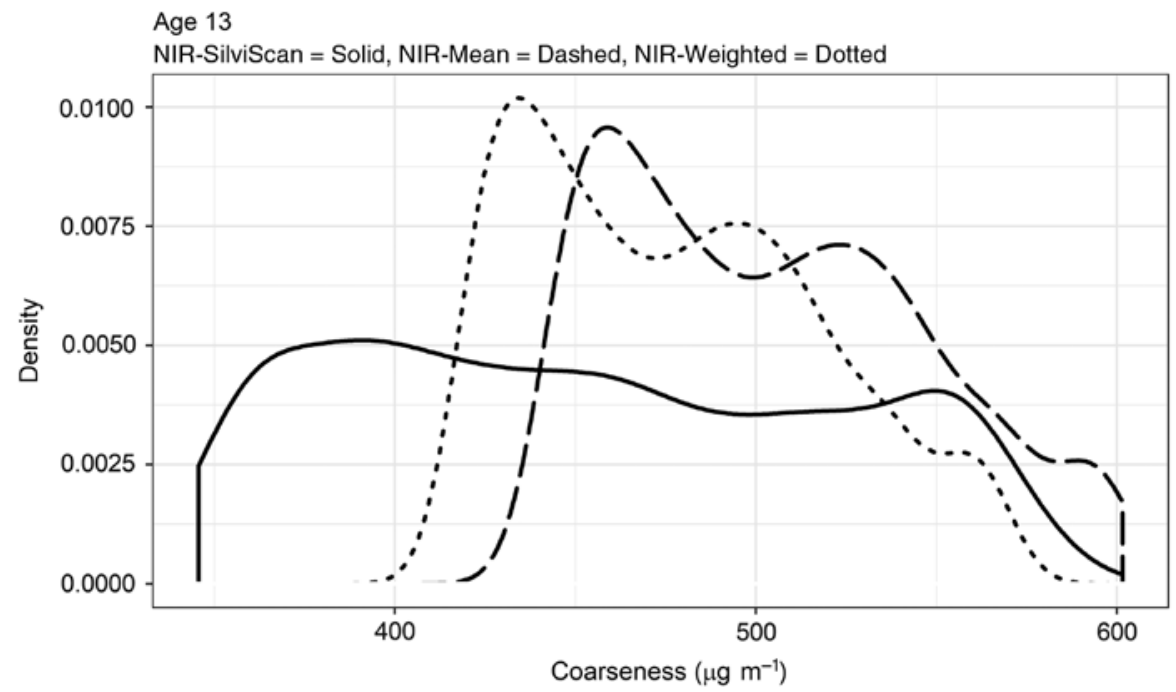

Figure 7: Probability (density) plots showing the variation of predicted coarseness for trees aged 13 years.

to predicted density as expected, given the adjustment done on the data in an attempt to correct for differences in coarseness that occur during pulping. Both the NIRMean and NIR-Weighted models had the same prediction statistics $\left(R^{2}=0.83,41.2 \mu \mathrm{g} \mathrm{m}^{-1}\right)$ as the NIR-SilviScan coarseness.

Table 2 provides a comparison of measured and predicted data at the whole-tree level. For both the 


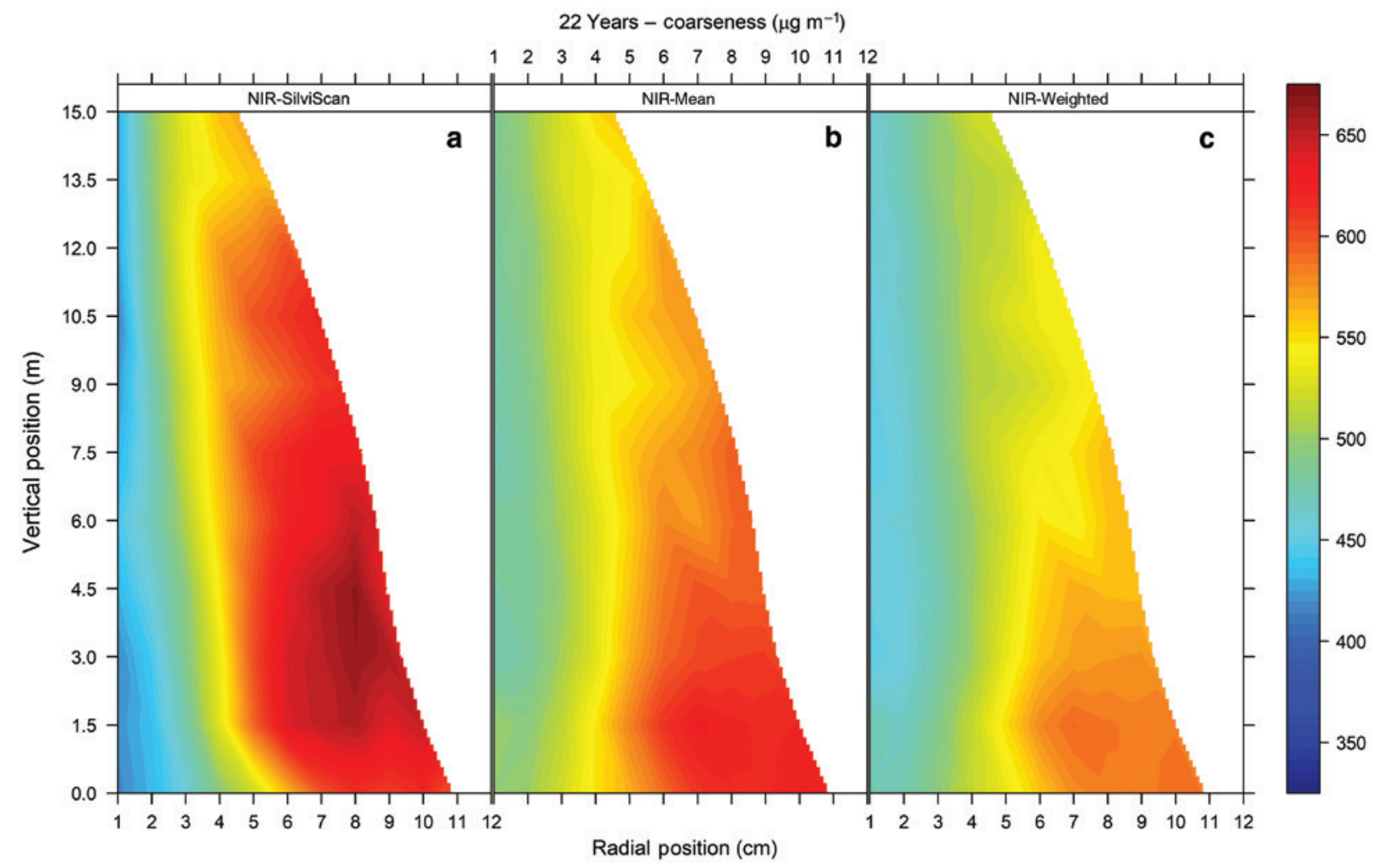

Figure 8: Maps showing within-tree variation of tracheid coarseness for loblolly pine trees aged 22 years.

Maps represent the average of 18 trees and were developed using Akima's interpolation and data provided by (a) spatial-specific ADD model (NIR-SilviScan), (b) interpolation-specific ADD model (NIR-Mean) and (c) interpolation-specific ADD model (NIR-Weighted).

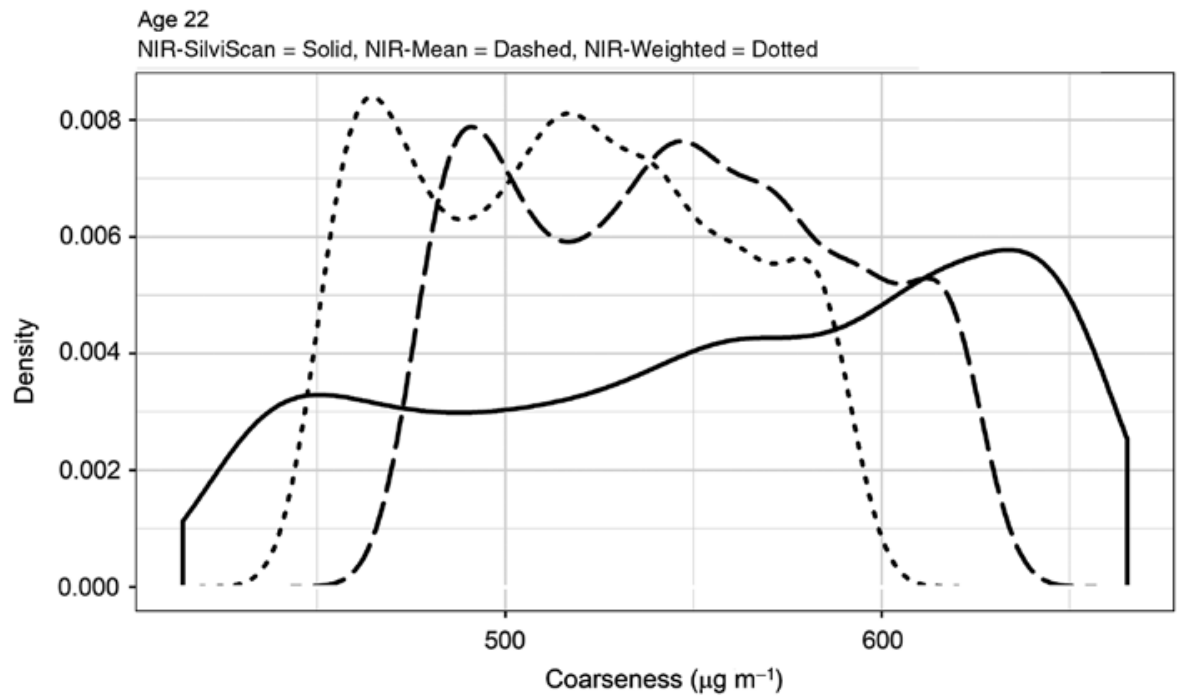

Figure 9: Probability (density) plots showing the variation of predicted coarseness for trees aged 22 years.

13- and 22-year-old trees, the NIR-Weighted model (524 and $584 \mathrm{~kg} \mathrm{~m}^{-3}$ ) yielded a closer prediction to the measured ADD (542 and $591 \mathrm{~kg} \mathrm{~m}^{-3}$ ) than the NIR-Mean model (576 and $637 \mathrm{~kg} \mathrm{~m}^{-3}$ ). Differences between measured whole-tree ADD and NIR-SilviScan ADD (570 and $605 \mathrm{~kg} \mathrm{~m}^{-3}$ ) were also observed and can be expected because the measurements are based on different principles, one gravimetric on whole-tree chips, the other X-ray densitometry on radial strips. For coarseness, again the NIR-Weighted model (495 and $535 \mu \mathrm{g} \mathrm{m}^{-1}$ ) was more similar to measured 

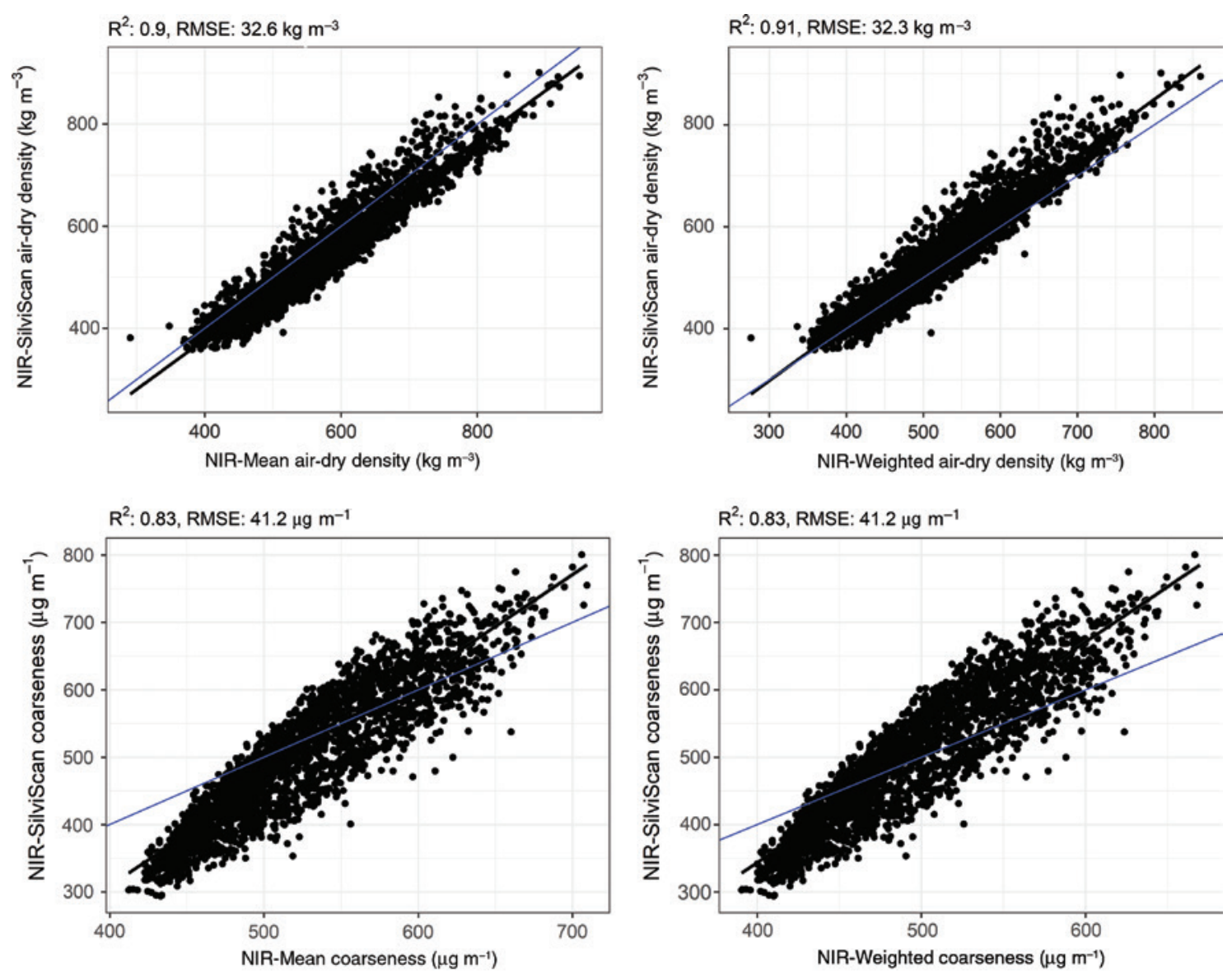

Figure 10: Plots of NIR-SilviScan-predicted density vs. NIR-Mean- and NIR-Weighted- predicted density and NIR-SilviScan-predicted coarseness vs. NIR-Mean- and NIR-Weighted-predicted coarseness.

The blue line represents the line of equivalence.

Table 2: Comparison between the mean measured and predicted whole-tree mean properties for density and coarseness for the 13- and 22-year-old trees.

\begin{tabular}{lrrrrr}
\hline & & & & Whole-tree means (18 trees) \\
\cline { 3 - 6 } Property & Age & Measured & NIR-SilviScan & NIR-Mean & NIR-Weighted \\
\hline Density $\left(\mathrm{kg} \mathrm{m}^{-3}\right)$ & 13 & 542 & 570 & 576 & 524 \\
& 22 & 591 & 605 & 637 & 584 \\
Coarseness $\left(\mu \mathrm{g} \mathrm{m}^{-1}\right)$ & 13 & 500 & 488 & 524 & 495 \\
& 22 & 545 & 588 & 566 & 535 \\
\hline
\end{tabular}

NIR, Near-infrared.

data (500 and $545 \mu^{-1} \mathrm{~g} \mathrm{~m}^{-1}$ ) than the NIR-Mean model (524 and $\left.566 \mu \mathrm{g} \mathrm{m}^{-1}\right)$. As expected, greater differences were observed for whole-tree vs. NIR-SilviScan coarseness ( 488 and $588 \mu \mathrm{g} \mathrm{m}^{-1}$ ) than what was observed for density, due to the variable impact pulping has on tracheid dimensions, and our use of the coarseness adjustment model developed for radiata pine. For the four sets of comparisons, the NIR-Weighted model was more accurate than the NIR-Mean model at estimating measured whole-tree values because weighted NIR spectra better represent the basal area of each NIR radial measurement within a disc.

Maps showing the within-tree variation of the ADD and coarseness for loblolly pine trees aged 13 and 22 years were developed using data provided by two different types of NIR-based models, referred to in this study as spatial-specific (NIR-SilviScan) and spatial-interpolated (NIR-Mean and NIR-Weighted). The two approaches fundamentally differ with the wood property data utilized 
measured at very different scales. For the spatial-specific model, the NIR and SilviScan data were measured in 10-mm increments, while for the spatial-interpolated models (one utilizing basal-area weighted average wholetree spectra, and the other arithmetic average wholetree spectra) the ADD and coarseness were measured on whole-tree composite samples.

ADD maps at both ages, obtained using the different NIR-based models, were compared and patterns of withintree variation were similar regardless of the models used. Minor differences were observed in the maps and were confirmed by probability plots; however, the magnitude of the differences were relatively small (Figures 3 and 5). Tracheid coarseness maps (Figures 6 and 8 ) were also very similar and for both properties and differences are not sufficient to change the interpretation of how ADD and tracheid coarseness vary within loblolly pine trees.

The result is highly significant for future studies of wood property variation within trees. It demonstrates that properties typically measured on whole-tree composite samples (for example, pulp yield, but in this study, ADD and tracheid coarseness) can be used to develop NIRbased whole-tree models that can be used to estimate properties utilizing spectra collected at much higher spatial resolution (in this study from radial strips in 10 -mm increments). Our findings also provide confidence that plots of within-sample variability based on NIR-HSI data (Thumm et al. 2010; Schimleck et al. 2018b), where whole-sample wood property data and average spectra are used to predict property variation at high pixel-bypixel resolution, give realistic representations of withinsample variability.

The comparative approach of whole-tree maps utilized in this study was only possible as NIR spectra were measured in 10-mm increments at multiple heights and radial positions (allowing whole-tree average spectra to be obtained for each tree), and we had wood properties measured at both a specific level (radial positions, $10-\mathrm{mm}$ increments) and the whole-tree level (on composite chips). If our approach were to be utilized in future studies of within-tree variation, NIR spectra would still need to be measured at multiple positions on radial samples and at multiple heights. For this study, preparation of samples and collection of NIR spectra (2569 in total) was a considerable undertaking, and now the equivalent amount of data can be quickly collected from radial strips or crosssectional discs using NIR-HSI.

This study compared tree maps of density adjusted to air-dry conditions measured from radial strips using SilviScan and X-ray densitometry, to density adjusted to air-dry conditions measured using water displacement from chips that were processed from whole trees. Also explored were maps of coarseness measured on dry samples using SilviScan, and coarseness measured on macerated pulp. The technique explored here illustrates that the spatial-interpolated method was successful for generating information on within-tree variation and the results can be applied to other properties that are commonly determined on whole-tree chips, including wood chemistry parameters such as pulp yield, as well as handsheet properties, provided a suitable NIR model can be calculated. The spatial-interpolated approach is not suitable for some properties such as MFA, due to the difficulty in obtaining these measurements on macerated tracheids as compared to the SilviScan system using X-ray diffraction.

\section{Conclusions}

Two different approaches for estimating wood properties based on NIR spectroscopy were compared by using NIR-predicted data to develop maps showing ADD and coarseness variation within loblolly pine trees aged 13 and 22 years. The first approach utilized data and NIR spectra measured in 10-mm increments and provided spatial-specific ADD and coarseness NIR models, while the second (spatial-interpolated) involved measuring a property on a broad-scale, in this study the whole tree, and calibrating this data against whole-tree NIR spectra. Whole-tree NIR spectra were obtained by averaging NIR spectra (arithmetic and weighted averages were determined) collected in 10-mm increments from radial strips at multiple heights. The spatial-specific model predicted $\mathrm{ADD}$ and coarseness at the equivalent scale $(10 \mathrm{~mm})$ while the spatial-interpolated model (whole tree) was used to predict ADD and coarseness at much higher resolution $(10 \mathrm{~mm})$. Maps of ADD and coarseness variation within loblolly pine, obtained using the different approaches, were comparable at both ages indicating that both approaches can be utilized to examine within-tree variation. Owing to improved accuracy, we recommend utilization of weighted NIR spectra for spatial-interpolated models. Spatial-interpolated models have a distinct advantage; being based on whole-tree samples, they greatly reduce the cost of wood property analysis and allow the development of maps for properties that are costly and difficult to measure, for example, pulp yield.

Acknowledgments: The authors thank the Wood Quality Consortium for collecting the Pinus taeda samples and for sample preparation. 
Author contributions: All the authors have accepted responsibility for the entire content of this submitted manuscript and approved submission.

Research funding: Support for this work was provided by the Georgia TIP ${ }^{3}$ program, the Wood Quality Consortium, the National Science Foundation (NSF) Center for Advanced Forest Systems (CAFS), and the NIFA McIntireStennis program (project 1006098). Support for this project is gratefully acknowledged.

Employment or leadership: None declared.

Honorarium: None declared.

\section{References}

Akima, H., Gebhardt, A. (2016) akima: Interpolation of irregularly and regularly spaced data. $\mathrm{R}$ package version 0.6-2. https:// CRAN.R-project.org/package=akima. Accessed January 15, 2019.

Burdon, R.D., Kibblewhite, R.P., Walker, J.C.F., Megraw, R.A., Evans, R., Cown, D.J. (2004) Juvenile versus mature wood: a new concept, orthogonal to corewood versus outerwood, with special reference to Pinus radiata and $P$. taeda. For. Sci. 50:399-415.

Burger, J., Gowen, A. (2011) Data handling in hyperspectral image analysis. Chemom. Intell. Lab. Syst. 108:13-22.

Çöpür, Y., Makkonen, H., Amidon, T.E. (2005) The prediction of pulp yield using selected fiber properties. Holzforschung 59 : 477-480.

Evans, R. (1994) Rapid measurement of the transverse dimensions of tracheids in radial wood sections from Pinus radiata. Holzforschung 48:168-172.

Evans, R. (1999) A variance approach to the X-ray diffractometric estimation of microfibril angle in wood. Appita J. 52:283.

Evans, R. (2006) Wood stiffness by X-ray diffractometry. In: Characterization of the Cellulosic Cell Wall. Eds. Stokke, D.D., Groom, L.H. Blackwell Publishing, Ames, IA. pp. 138-146.

Evans, R., Stringer, S., Kibblewhite, R.P. (2000) Variation of microfibril angle, density and fibre orientation in twenty-nine Eucalyptus nitens trees. Appita J. 53:450-457.

Glass, S.V., Zelinka, S.L. (2010) Moisture relations and physical properties of wood. In: Wood Handbook. Gen Tech Rep FPL-GTR-190. Ed. Ross, R.J. U.S. Department of Agriculture, Forest Service, Forest Products Laboratory, Madison, WI. pp 4-1-4-19.

Jordan, L., Clark, A., Schimleck, L.R., Hall, D.B., Daniels, R.F. (2008) Regional variation in wood specific gravity of planted loblolly pine in the United States. Can. J. For. Res. 38:698-710.

Kibblewhite, R.P., Evans, R. (2001) Dimensional relationships among radiata pine wood tracheid, and chemical and TMP pulp fibres. Appita J. 54:297-303.

Lundqvist, S.-O., Ekenstedt, F., Hedenberg, O., Twaddle, A. (2005) Wood and fiber properties of loblolly pine in the southeast USA. Variations and prediction models. In: Fifth Workshop, Connection Between Forest Resources and Wood Quality: Modelling Approaches and Simulation Software, Waiheke Island Resort, New Zealand, 20-27 November, 2005.
Ma, T., Inagaki, T., Tsuchikawa, S. (2017) Calibration of SilviScan data of Cryptomeria japonica wood concerning density and microfibril angles with NIR hyperspectral imaging with high spatial resolution. Holzforschung 71:341-347.

Ma, T., Inagaki, T., Tsuchikawa, S. (2018) Non-destructive evaluation of wood stiffness and fiber coarseness, derived from SilviScan data, via near infrared hyperspectral imaging. J. Near Infrared Spectrosc. 26:398-405.

Mevik, B.-H., Wehrens, R., Hovde Liland, K. (2013) pls: Partial Least Squares and Principal Component Regression. R package version 2.4-3. http://CRAN.R-project.org/package=pls.

Mora, C.R., Schimleck, L.R. (2009) Determination of within-tree variation of Pinus taeda wood properties by near infrared spectroscopy. Part 2: whole-tree wood property maps. Appita J. 62:232.

Nabavi, M., Dahlen, J., Schimleck, L., Eberhardt, T.L., Montes, C. (2018) Regional calibration models for predicting loblolly pine tracheid properties using near-infrared spectroscopy. Wood Sci. Technol. 52:445-463.

Nychka, D., Furrer, R., Paige, J., Sain, S. (2015) fields: Tools for spatial data. R package version 8.10. http://CRAN.R-project.org/ package=fields. Accessed January 15, 2019.

R Core Team. R: A Language and Environment for Statistical Computing. R Foundation for Statistical Computing, Vienna, Austria, 2018.

RStudio. RStudio: Integrated Development Environment for R. Boston, MA, USA, 2018.

Sarkar, D. Lattice: Multivariate Data Visualization with R. Springer, New York, USA, 2008.

Scallan, A.M., Green, H.V. (1975) The effect of pulping upon the dimensions of wood tracheids. Wood Fiber Sci. 7:226-233.

Schimleck, L.R., Michell, A.J. (1998) Determination of within-tree variation of kraft pulp yield using near-infrared spectroscopy. TAPPI J. 81:229-236.

Schimleck, L.R., Evans, R., Ilic, J. (2001) Estimation of Eucalyptus delegatensis wood properties by near infrared spectroscopy. Can. J. For. Res. 31:1671-1675.

Schimleck, L.R., Evans, R. (2004) Estimation of Pinus radiata D. Don tracheid morphological characteristics by near infrared spectroscopy. Holzforschung 58:66-73.

Schimleck, L.R., Mora, C.R., Jordan, L., White, D.E., Courchene, C.E., Purnell, R.C. (2009) Determination of within-tree variation of Pinus taeda wood properties by near infrared spectroscopy. Part 1: development of multiple height calibrations. Appita J. 62:130-136.

Schimleck, L., Antony, F., Mora, C., Dahlen, J. (2018a) Comparison of whole-tree wood property maps for 13- and 22-year-old loblolly pine. Forests 9:287.

Schimleck, L., Dahlen, J., Yoon, S.-C., Lawrence, K., Jones, P.D. (2018b) Prediction of Douglas-fir lumber properties: comparison between a benchtop near infrared spectrometer and hyperspectral imaging system. Appl. Sci. 8:2602.

Signal Developers (2013). Signal: Signal Processing. URL: http://rforge.r-project.org/projects/signal/.

Thumm, A., Riddell, M., Nanayakkara, B., Harrington, J., Meder, R. (2010) Near infrared hyperspectral imaging applied to mapping chemical composition in wood samples. J. Near Infrared Spectrosc. 18:507-515.

Thumm, A., Riddell, M., Nanayakkara, B., Harrington, J., Meder, R. (2016) Mapping within-stem variation of chemical composition 
by near infrared hyperspectral imaging. J. Near Infrared Spectrosc. 24:605-616.

Wickham, H., Francois, R. (2016) dplyr: A grammar of data manipulation. $R$ package version 0.4.3. https://CRAN.R-project.org/ package $=$ dplyr. Accessed January 15, 2019.

Wickham, H., Rstudio. (2017) tidyverse: Easily install and load the 'tidyverse' R package version 1.2.1. https://cran.r-project.org/ web/packages/tidyverse/index.html.

White, D.E., Courchene, C., McDonough, T., Schimleck, L., Jones, D., Peter, G., Purnell, R., Goyal, G. (2009) Effects of specific gravity and wood chemical content on the pulp yield of loblolly pine. TAPPI J. 92:29.

White, D.E., Courchene, C., McDonough, T., Schimleck, L., Peter, G., Rakestraw, J., Goyal, G. (2011) Effects of loblolly pine wood and pulp properties on sheet characteristics. TAPPI J. 10:36-42.

Williams, P.C., Sobering, D.C. (1993) Comparison of commercial near infrared transmittance and reflectance instruments for analysis of whole grains and seeds. J. Near Infrared Spectrosc. 1:8. 